Physica B 171 (1991) 175-184

North-Holland

\title{
Electron spectroscopy of strongly correlated systems
}

\author{
J.W. Allen \\ The Randall Laboratory of Physics, University of Michigan, Ann Arbor, MI 48109-1120, USA \\ Invited paper.
}

\begin{abstract}
An overview is given of current electron spectroscopy studies of the single-particle electronic structure of strongly correlated systems. Specific topics include (i) the Ce $4 \mathrm{f}$ spectrum of $\mathrm{CeCu}_{2} \mathrm{Si}_{2}$, (ii) the $5 \mathrm{f}$ spectrum of $\mathrm{Y}_{1-x} \mathrm{U}_{x} \mathrm{Pd}_{3}$, and (iii) the doped metallic state of high-temperature superconductor materials, for which it is argued that in the Luttinger liquid scenario, the Fermi energy of the metal should lie in the parent insulator's gap, as observed experimentally.
\end{abstract}

\section{Introduction}

This paper presents an overview of current photoemission and inverse photoemission studies of the single-particle electronic structure of strongly correlated systems. Strongly correlated systems are found in the group of materials where the valence and conduction bandwidths are less than the magnitude of Coulomb interactions between electrons in these states [1]. In the main, this situation occurs for the $3 \mathrm{~d}$ electrons of transition metal compounds, the $4 \mathrm{f}$ electrons of rare earth elements and compounds, and the $5 f$ electrons of actinide elements and compounds. Implicit in the idea of a strongly correlated system is that many-body effects are essential in a description of the electronic structure. In this case, it is important to have in mind a definition of the single-particle electronic structure that is sufficiently general to include these effects. The appropriate definition is the spectrum to remove and add an electron, i.e., the electron ionization and affinity spectra. The theoretical response function is known as the single-particle Green's function $G$. No mathematics is required to appreciate that in a system of non-interacting electrons in a solid this spectrum is exactly the one-electron spectrum of a traditional band description. For a system of interacting electrons, the spectrum is more complex, with broadened, shifted or entirely new peaks.
The principle experimental techniques for determining the single particle electronic structure are photoemission spectroscopy (PES) and inverse photoemission spectroscopy (IPES), for measuring the ionization and affinity spectra, respectively. When IPES is performed at X-ray energies, it is generally called Bremsstrahlung isochromat spectroscopy (BIS). These spectroscopies produce final states with one extra hole or electron via photon absorption and electron emission, or electron injection and photon emission, respectively. In making the connection to the theoretical response function it is necessary to assume that the sudden approximation holds for the photon event, i.e., that the outgoing or incoming electron in PES or BIS, respectively, has enough kinetic energy that its interaction with the system after or before the photon event is negligible. Then the photon event can be characterized by a cross section $\sigma$. In angleresolved photoemission spectroscopy (ARPES) electrons are collected in a well-defined direction rclative to an oriented single crystal surface, which allows the selection of electrons of a particular crystal momentum $k$. IPES can also be performed in the angle-resolved mode.

The emission from a particular kind of electronic state ' $i$ ' can often be identified because its cross section has a particular photon energy $(h \nu)$ dependence $\sigma_{i}(h \nu)$. Nearly all PES measurements have utilized this dependence to enhance, 
and sometimes to extract the spectral features associated with the narrow band electrons of interest. For $\mathrm{Ce} 4 \mathrm{f}, \mathrm{U} 5 \mathrm{f}$ and $\mathrm{Cu}$ or Ni $3 \mathrm{~d}$ states, the cross section has a resonance for $h \nu$ near the $\mathrm{Ce} 4 \mathrm{~d}, \mathrm{U} 5 \mathrm{~d}$ and $\mathrm{Cu}$ or $\mathrm{Ni} 3 \mathrm{p}$, absorption edges, around 120,100 and $70 \mathrm{eV}$, respectively, involving a mechanism described elsewhere $[2,3]$. Exploiting these resonances is known as resonant photoemission spectroscopy (RESPES) [3] and is performed with synchrotron radiation. Recent claims [4] that RESPES provides misleading results for uranium systems appear to have been refuted entirely [5].

The remainder of this paper discusses, in turn, current work for rare earths, actinides and $\mathrm{Cu}-\mathrm{O}$ high-temperature superconductor (HTSC) materials. For the most part, the paper focuses on developments not discussed in an overview paper on heavy-fermion spectroscopy written for the 1988 ICCF6 conference [6], although there is some overlap. The rare earth section of the paper is a shortened version of the discussion in a more recent review [7] written for a 1989 conference on synchrotron spectroscopy, and is included here for completeness. The present paper differs from both of the previous overviews by including new $B I S$ data for $\mathrm{Y}_{1-x} \mathrm{U}_{x} \mathrm{Pd}_{3}$, and a discussion of the metallic state of HTSC materials.

\section{Rare earth systems}

\subsection{Anderson impurity Hamiltonian}

For cerium and ytterbium materials, a coherent account of both the 4f PES/BIS spectra and certain ground-state properties has been obtained using the degenerate impurity Anderson Hamiltonian $[2,3,8-11]$. This Hamiltonian is a model for an $N_{\mathrm{f}}$-fold degenerate local orbital, denoted here as f, hybridized to a band. It contains several charge fluctuation energy scales, $U_{\mathrm{ff}}, \epsilon_{\mathrm{f}}$ and $\rho N_{\mathrm{f}} V^{2}$, which are, respectively, the Coulomb repulsion between two f electrons, the $\mathrm{f}$ binding energy relative to the Fermi level $E_{\mathrm{F}}$, and the hybridization width involving hybridization matrix element $V$ of the $f$ state to the conduction electrons having density of states $\rho$. Other energy scales for the f electrons can also be included in the model, such as spin-orbit, or crystal field splitting of the $f$ degeneracy. This model has an emergent energy scale, $k_{\mathrm{B}} T_{\mathrm{K}}$ which characterizes spin fluctuations and its inverse is proportional to the $T$-linear specific coefficient $\gamma$ and the DC magnetic susceptibility $\chi(0) . T_{\mathrm{K}}$ is called the Kondo temperature, and also controls the width and weight of a Fermi-level peak, the Kondo resonance, in the BIS/PES spectrum. Small $T_{\mathrm{K}}$ means small weight in the resonance, a large value of $\gamma$, and a large value of $\chi(0)$.

Other $4 \mathrm{f}$ energy scales included in the model, such as spin-orbit or crystal field splittings, produce additional structure on both the PES and BIS sides of the Kondo resonance, and provide additional weight near $E_{\mathrm{F}}$ relative to that which would occur for the same $T_{\mathrm{K}}$. These details of the resonance are observed for both $\mathrm{Ce}$ [12] and $\mathrm{Yb}$ [11] materials. In principle, the resonance should also show structure associated with lattice effects neglected in the impurity approach. A theoretical attack on this problem $[13,14]$ is to calculate the band structure in the local density approximation (LDA), to replace the LDA phase shifts for the $f$ electrons by phase shifts appropriate to the Kondo resonance, and then to calculate a renormalized band structure. This approach has been applied to deHaas-van Alphen data, but the renormalized band structure has yet to be observed in PES or IPES.

It has also been possible to obtain a good description $[2,9]$ of the spectra obtained in X-ray photoemission spectroscopy (XPS) of cerium 3dcore levels, by augmenting the impurity Anderson Hamiltonian with an interaction term of magnitude $U_{\mathrm{fc}}$ which increases the binding energy $\epsilon_{\mathrm{f}}$ in the presence of a core hole. It is found that the $4 \mathrm{f}$ PES/BIS spectra and the $3 \mathrm{~d}$ XPS spectra can be described with very similar values of Hamiltonian charge fluctuation parameters, and that the low-energy properties calculated with these parameters are in reasonable agreement with experiment if $T_{\mathrm{K}}$ is not exceptionally small. 


\subsection{Origin of heavy-fermion behavior in $\mathrm{CeCu}_{2} \mathrm{Si}_{2}$}

Current efforts for $4 \mathrm{f}$ electrons are directed at finding the quantitative limits of the impurity approach by making the model as realistic as possible. One step in this direction is to use an energy dependent $V(E)$ calculated within the LDA explicitly for the material under study. A realistic $V(E)$ is especially important for obtaining good fits to PES spectra, and also provides insight into the electronic structure origin of the ground state properties of interest. In fitting spectra, it is found that scaling the magnitude of $V(E)$ is necessary to obtain good fits, or in some cases, to reproduce the magnetic susceptibility. Also, the values of $\epsilon_{\mathrm{f}}$ and $U_{\mathrm{ff}}$ are taken as adjustable. However, it should be pointed out in passing that the LDA and related techniques can be used to calculate these two energy parameters, essentially from first principles $[15,16,17]$. Thus the combination of the many-body model and LDA calculations provides the possibility of a predictive, microscopic many-body theory of both the spectral and ground state properties of the $4 \mathrm{f}$ electrons at the level of the impurity model.

$\mathrm{CeCu}_{2} \mathrm{Si}_{2}$, the original heavy-fermion superconductor material $[18,19]$, has been studied recently using a calculated $V(E)$. Details of the calculation, and the fitting of spectra are given elsewhere $[17,20]$. Figure 1 shows that structure in the partial densities of state of the $\mathrm{Cu}$ $\mathrm{d}$ electrons and of the Si $\mathrm{p}$ electrons are reflected in $|V(E)|^{2}$. For $-6 \mathrm{eV} \leqslant E \leqslant-3 \mathrm{eV}$, coupling to the $\mathrm{Cu} \mathrm{d}$ states dominates. Coupling to the $\mathrm{Si}$ p-states dominates the region near $E_{\mathrm{F}}$, so it is most important in determining ground state properties, whereas $\mathrm{Ce} 4 \mathrm{f}$ spectral features are determined by both. The data points in panels (a) through (c) of fig. 2 show the experimental $4 \mathrm{f}$ BIS, 4f PES and 3d XPS spectra, and the solid lines the corresponding theoretical spectra, which have been broadened to simulate the experimental resolution. The PES spectrum was extracted from the RESPES data of ref. [21] by methods described in ref. [2]. In general the theory provides a very good description of the

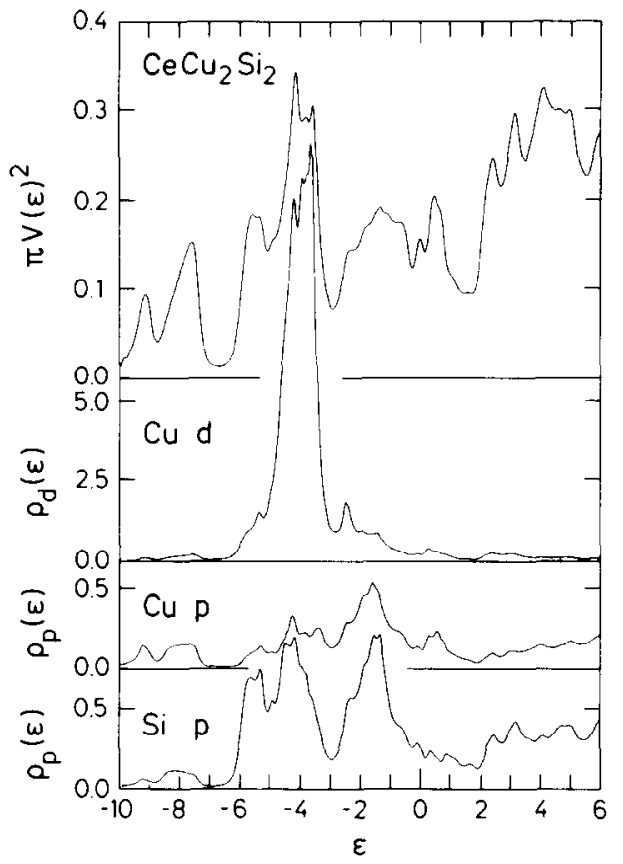

Fig. 1. LDA results for $\mathrm{CeCu}_{2} \mathrm{Si}_{2}$. Top panel: the square of the magnitude of the $4 \mathrm{f}$ band states hybridization matrix element of the impurity Anderson model. Middle and bottom panels: the projected $\mathrm{Cu} 3 \mathrm{~d}$ and Si $3 \mathrm{p}$ densities of states, respectively, broadened by a Lorentzian with the full width $0.14 \mathrm{eV}$.

experimental data. For the $3 \mathrm{~d}$ spectrum the excellent fit of the least binding energy spin-orbit component is not maintained in the higher-energy component because the theory does not include inelastic loss features present for binding energies greater than $890 \mathrm{eV}$. The model parameters for the fits are $\epsilon=-2.4 \mathrm{eV}, U_{\mathrm{ff}}=7.27 \mathrm{eV}$ and $U_{\mathrm{fc}}=11.5 \mathrm{eV}$. In addition it has been necessary to reduce the magnitude of the theory $V(E)$ by a factor 0.67 . The source of this renormalization is unknown, but a possible reason is that the LDA has an unphysical interaction of the f electron with itself, which would give $4 \mathrm{f}$ wavefunctions that are too extended.

Both the BIS and PES spectra show very little weight in the Kondo resonance, reflecting the fact that the ground-state composition, $1.1 \% 4 \mathrm{f}^{0}$, $95.6 \% 4 \mathrm{f}^{1}$, and $3.3 \% 4 \mathrm{f}^{2}$, is near integer valence, and $T_{\mathrm{K}}$ is very small. It appears generally to be the case [2] that small $T_{\mathrm{K}}$, and hence heavy- 


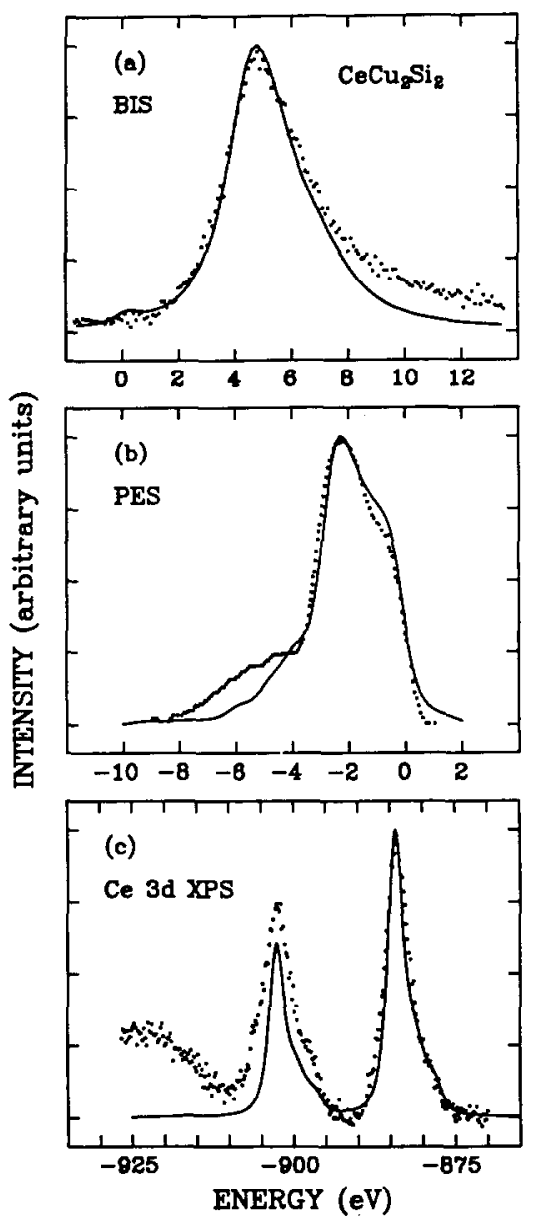

Fig. 2. Comparison of the spectrum calculated using the impurity Anderson model (solid lines) with the experimental spectrum (dots) for (a) 4f BIS, (b) 4f PES and (c) Ce 3d XPS in $\mathrm{CeCu}_{2} \mathrm{Si}_{2}$. The $4 \mathrm{f}$ PES spectrum was extracted from the data in ref. [21].

fermion behavior, results when hybridization near $E_{\mathrm{F}}$ is to $\mathrm{s} / \mathrm{p}$ states rather than to d states. The spectra of fig. 2 have been calculated in a model which includes only spin-orbit splitting of the $4 f^{1}$ state. To calculate $\chi(0)$ it is very important to include also the crystal field splitting [22], and this has been done to lowest order in the inverse degeneracy theory [9]. For the $V(E)$ determined spectroscopically, the resulting $\chi(0)$ is larger than the experimental one by 3 to 4 orders of magnitude. To obtain the experimental value it is necessary to increase the spectroscopic $V(E)$ by a factor of about 1.2 . That a relatively small correction in $V(E)$ is required to remove such a large discrepancy in $\chi(0)$ is because $T_{\mathrm{K}}$ depends exponentially on the model parameters in the heavy-fermion (small $T_{\mathrm{K}}$ ) regime of the model. It has been found that, although $V(E)$ varies considerably for different $f$ orbitals, the neglect of this dependence is not the origin of the need for renormalization. [23] A possible explanation discussed in detail elsewhere [24] is that the Coulomb interaction between the $f$ electrons and the conduction electrons, which is not included explicitly in the model, causes $V(E)$ to be different for spectroscopic and thermodynamic properties. In the current theory of this effect, it is large only for materials with very small $T_{\mathrm{K}}$.

\section{Uranium systems}

\subsection{Introduction}

In general, the $5 f$ spectra of metallic uranium materials [3,25] do not resemble the 4f spectra of rare earth materials, especially in having large amounts of weight around $E_{\mathrm{F}}$. This is true even for heavy-fermion materials, which, in the impurity Anderson model, would have small $T_{\mathrm{K}}$ and therefore small weight at $E_{\mathrm{F}}$. Onc approach to interpreting uranium spectra has been to compare with LDA calculations, as summarized elsewhere $[3,6]$. Another approach is to study the effects of diluting the uranium with a chemically similar element lacking 5 f electrons, such as yttrium. The goal is to distinguish single site from lattice effects in at least the large energy scale features of the $5 \mathrm{f}$ spectra, with the hope of deciding whether an Anderson impurity model could serve as a useful starting point for a theoretical description. Various dilution studies are described in ref. [6]. In general the spectra obtained in PES and BIS have been entirely, or nearly independent of $x$.

\subsection{Dilution studies of $Y_{1-x} U_{x} P d_{3}$}

The dilution system $\mathrm{Y}_{1-x} \mathrm{U}_{x} \mathrm{Pd}_{3}$ is interesting because it does not follow the trends just de- 
scribed. The $x=1$ compound is unusual in that the PES/BIS spectrum shows a gap around $E_{\mathrm{F}}$. The usual interpretation $[26-28]$ is that the PES and BIS spectra consist of $5 f^{2} \rightarrow 5 f^{1}$ and $5 f^{2} \rightarrow 5 f^{3}$ transitions, respectively, and that the gap measures $U_{\mathrm{ff}}$. As reported recently [29], the 5f PES spectrum, deduced using RESPES, changes significantly with dilution. As shown in fig. 3, the spectrum narrows and moves toward $E_{\mathrm{F}}$ as $x$ decreases from 1 to 0.1 . A large fraction of the shift occurs for $x$ between 0.1 and 0.5 , and so the shift does not seem to be especially correlated with a crystal structure change that takes place between $x=0.5$ and $x=0.9$. A figure from ref. [29] shows that the spectrum for $x=0.02$ is essentially identical to that for $x=0.1$ within experimental uncertainty due to noise and resolution. An interpretation advanced for this result within the usual model for $\mathrm{UPd}_{3}$ is that $\mathrm{U}^{4+}$, i.e. $5 \mathrm{f}^{2}$, is replaced by $\mathrm{Y}^{3+}$. The number of conduction electrons per unit cell will then decrease by 1 as $x$ changes from 1 to 0 . Band calculations for $\mathrm{YPd}_{3}[30]$ indicate that the den- sity of conduction electron states within the interval of about $1.0 \mathrm{eV}$ above and below $E_{\mathrm{F}}$ is roughly 1.0 electrons/eV-unit cell, implying that $E_{\mathrm{F}}$ will decrease by about $1 \mathrm{eV}$ as $x$ changes from 1 to 0 , as observed. Consistent with this interpretation is that the Pd $4 \mathrm{~d}$ valence band emission shifts toward $E_{\mathrm{F}}$ by a comparable amount.

Subsequent core level XPS and BIS studies have been made [31]. The new data are generally consistent with the idea of a shifting $E_{\mathrm{F}}$, but also suggest that a more detailed picture will be required, e.g., for the charge transfer in the alloy, and for the $\mathrm{U} 5 \mathrm{f}$ electronic structure. It is found, e.g., that the Pd and Y $3 \mathrm{~d}$ core levels shift toward $E_{\mathrm{F}}$, but by different amounts of about 1.1 and $0.7 \mathrm{eV}$, respectively, while the $\mathrm{U}$ 4f core levels display a more complex behavior which will be reported elsewhere [31]. Figure 3 shows the BIS spectrum for various $x$. Spectra for $x=0.9$ and $x=0.3$ fit smoothly in the sequence, but are not shown for clarity. Antibonding states of mixed $\mathrm{Y} / \mathrm{U} 5 \mathrm{~d}$ and $\mathrm{Pd} 4 \mathrm{~d}$ character $[30,32]$ make a substantial contribution to the

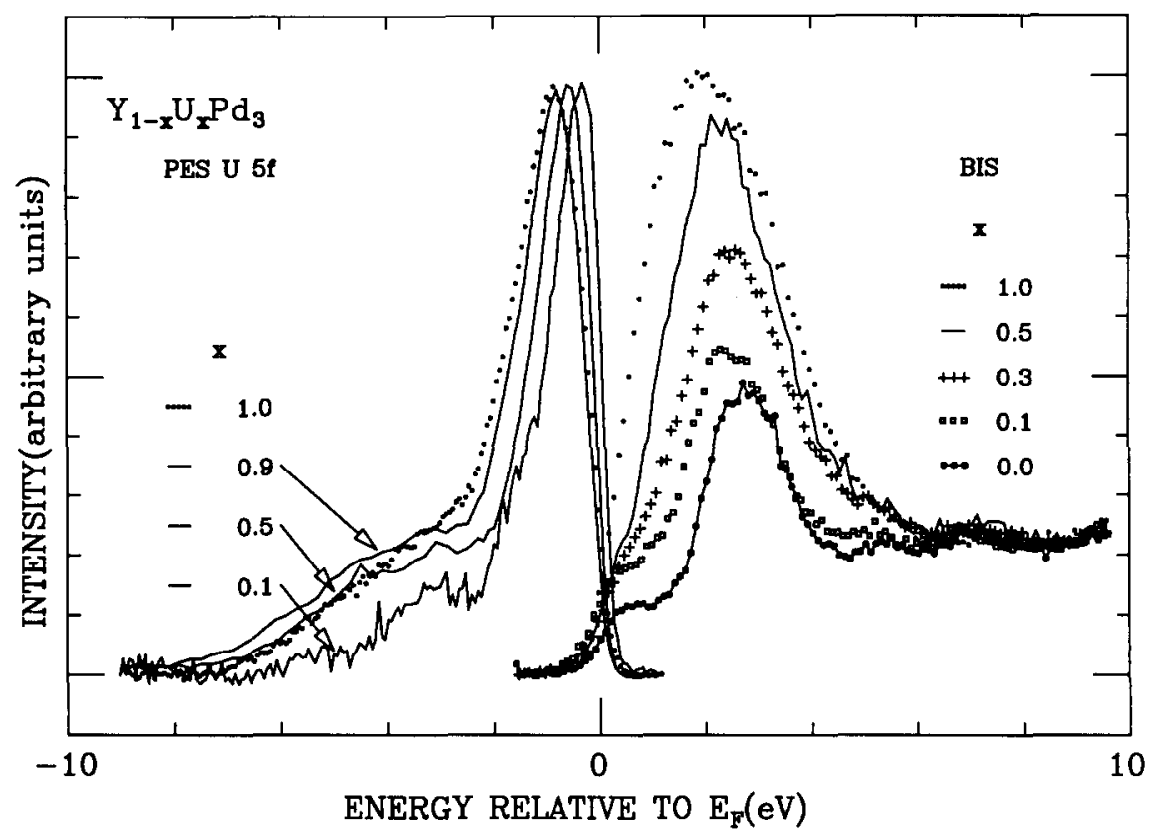

Fig. 3. PES/BIS spectra for $\mathrm{U}_{x} \mathrm{Y}_{1-x} \mathrm{Pd}_{3}$. Below $E_{\mathrm{F}}$ : the uranium $5 \mathrm{f}$ spectral weight for $x=0.1,0.5,0.9$ and 1.0 determined using RESPES. A figure in ref. [29] shows that the PES spectrum for $x=0.02$ is essentially identical to that for $x=0.1$ within experimental uncertainty. Above $E_{\mathrm{F}}: h \nu=1486.6 \mathrm{eV}$ BIS data for $x=1.0,0.5,0.3,0.1$, and 0 . Spectra for $x=0.9$ and 0.03 are not shown for clarity, but fit smoothly in the progression. 
total spectrum, as shown by the data for $x=0$. Data analysis to extract the U 5 f BIS spectrum is now in progress, and it is planned to attempt a fit of the $\mathrm{U} 5 \mathrm{f}$ and $4 \mathrm{f}$ spectra using the impurity Anderson model. In such a model, it is expected that $T_{\mathrm{K}}$ will change as the separation between the $5 f$ peak and $E_{\mathrm{F}}$ changes, and as the hybridization strength $V$ changes. Thus the transport and other ground state properties are expected to vary strongly with $x$, and it is hoped to correlate the spectroscopic results with the results of transport measurements now in progress in M.B. Maple's laboratory at the University of California at San Diego.

\section{Metallic state of the high-temperature superconductor $\mathrm{Cu}-\mathrm{O}$ materials}

\subsection{Introduction}

The normal metallic state of the $\mathrm{Cu}-\mathrm{O}$ hightemperature superconductor (HTSC) materials is achieved by doping parent insulating antiferromagnetic (AF) materials. It is generally agreed that a Coulomb interaction $\mathrm{U}$ between $\mathrm{Cu} 3 \mathrm{~d}$ electrons on the same site is essential in producing the insulating state of the parent materials, which are predicted by band theory to be metals. The simplest theoretical paradigm for this correlated insulator state is that of the one-band Hubbard model at half filling, and the experimental paradigm is $\mathrm{NiO}$ [33-35]. Current issues are the description of the electronic structure of such insulators, and the nature of the metal produced by doping them away from half filling. The following discussion describes work directed at these issues for the HISC materials and for $\mathrm{NiO}$.

\subsection{The insulator spectrum}

The data points of fig. 4, taken from refs. $[36,37]$ show angle-integrated RESPES spectra for the electron-doped system $[38,39]$ $\mathrm{Nd}_{2-x} \mathrm{Ce}_{x} \mathrm{CuO}_{4}$, for $x=0$ and 0.15 . The main features of the $x=0$ insulator spectrum are typical for other HTSC materials. There is a large weakly structured 'main band' and weaker 'satellite' features between -8 and $-16 \mathrm{eV}$. The sharp increase with $h \nu$ in the intensity of the satellite features shows that they have $\mathrm{Cu} 3 \mathrm{~d}$ character. Band theory predicts no $3 \mathrm{~d}$ weight in this energy range.

The top solid line of fig. 4 shows the $\mathrm{Cu} 3 \mathrm{~d}$ spectrum calculated for $\mathrm{Nd}_{2} \mathrm{CuO}_{4}$ in an impurity Anderson model [36] for the correlated $3 \mathrm{~d}$ electrons of a single $\mathrm{Cu}$ site, hybridized to the $\mathrm{O} 2 \mathrm{p}$ bands. The impurity ground state is a linear combination of $3 \mathrm{~d}^{9}$ and $3 \mathrm{~d}^{10} \mathrm{~L}{ }^{1}$ configurations. $\mathrm{L}^{-1}$ means a hole, having $\mathrm{d}$ symmetry around a $\mathrm{Cu}$ site, in an otherwise full $\mathrm{O} 2 \mathrm{p}$ band. The low binding energy states of the electron removal spectrum are mainly lincar combinations of $3 \mathrm{~d}^{4} \mathrm{~L}^{-1}$ and $3 \mathrm{~d}^{10} \mathrm{~L}^{-2}$, while the higher lying 'satellite' states are mainly of $3 \mathrm{~d}^{8}$ type. The dashed lines show the atomic $3 \mathrm{~d}^{8}$ multiplets, which lie at higher binding energy due to the large $U$. The hybridization with the low-lying states pushes $3 \mathrm{~d}^{8}$ weight to even higher binding energy and also mixes it into the low-lying states. As found in earlier work $[40,41]$, in the ${ }^{1} A_{1 g}$ symmetry, the hybridization can push out a bound state, the 'local singlet', above the valence band, which is the peak at lowest binding energy. The calculation agrees well with experiment in the satellite region, and has the same general features as the main band, but the weight of the local singlet is much larger than in experiment.

How good is the impurity description of the single particle electronic structure of the insulator? On the positive side, it serves to show the correlated character of the insulator. It also shows that the band gap is actually of charge transfer type $[42,43]$, which is important in giving meaning to a one-band Hubbard model applied to the system $[42,44,45]$. However, recent ARPES experiments for $\mathrm{NiO}$ strongly suggest that lattice effects neglected in the impurity description of the insulator can be observed [46] The main band features show strong dependences on emission angle, and on the photon energy and polarization. Features of oxygen $2 p$ character are in good agreement with the results of a band calculation, including the effect of $\mathrm{AF}$ order. Also the peaks with the smallest binding 
energies, which correspond to $3 \mathrm{~d}$ bands in the LDA, and to the $3 d^{8} \mathrm{~L}^{-1}$ and $3 \mathrm{~d}^{9} \mathrm{~L}^{-2}$ final states in the impurity model, display measureable dispersion, of order a few tenths of an $\mathrm{eV}$. For the HTSC materials, ARPES studies of the main band also suggest the importance of lattice effects $[47,48]$. These studies were done in the metallic state, but, as emphasized in section 4.3, doping often has little effect on the main band. The band structures of the HTSC materials are so complex that the extent of agreement with the ARPES data is difficult to judge, and in any case there is currently no theory which describes both the satellite and the lattice aspects of the insulator spectrum. A point often overlooked is that for the wavefunction implied by band theory, the least binding energy parts of the PES spectrum of $\mathrm{NiO}$ and the copper oxide superconductors should resonant strongly rather than weakly, because the $3 \mathrm{~d}$ content is very large $[3,49]$.

\subsection{The near- $E_{\mathrm{F}}$ states of the metal}

A result of great importance shown by current data is that the chemical potential of the doped metal lies in the insulator gap. With doping, it does not move into states already present in the insulator, and does not jump across the gap if the doping is changed from holes to electrons [37]. This result makes it unlikely that the $E_{\mathrm{r}}$ states of the metal are literally and simply the local singlet predicted for the insulator. Figure 4 shows PES data from ref. [37] for $\mathrm{Nd}_{2-x} \mathrm{Ce}_{x} \mathrm{CuO}_{4}$ for the metallic state with $x=$ 0.15 . The main-band features are essentially unchanged from those of the insulator, although the satellite is somewhat weaker. As highlighted in the inset, $E_{\mathrm{F}}$ lies in the insulator gap, as does the emission in the first $0.5 \mathrm{eV}$ below $E_{\mathrm{F}}$. Early PES data [50] show that the same occurs for the hole doped system $\mathrm{La}_{2-x} \mathrm{Sr}_{x} \mathrm{CuO}_{4}$, and that $E_{\mathrm{F}}$ is roughly the same for the two systems. Other workers have also noted and discussed this aspect of the near- $E_{\mathrm{F}}$ emission in $\mathrm{Nd}_{2-x} \mathrm{Ce}_{x} \mathrm{CuO}_{4}$ $[51,52]$ and $\mathrm{Bi}_{2} \mathrm{Sr}_{2} \mathrm{Ca}_{1-x} \mathrm{Y}_{x} \mathrm{Cu}_{2} \mathrm{O}_{8}[53,54]$.

The discussion of ref. [37] emphasizes the simultaneous consideration of hole and electron

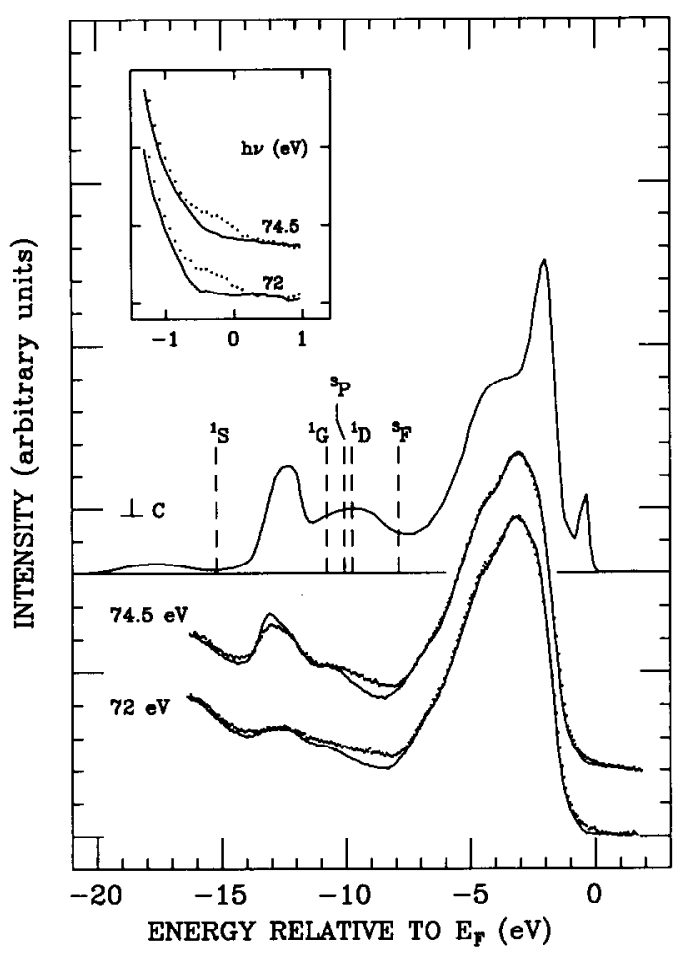

Fig. 4. Top curve: Calculated uff-resonance $\mathrm{Nd}_{2} \mathrm{CuO}_{4} \mathrm{Cu} 3 \mathrm{~d}$ PES spectrum in the Anderson impurity model. Other curves: valence band RESPES spectra for ceramic samples of $\mathrm{Nd}_{2-x} \mathrm{Ce}_{x} \mathrm{CuO}_{4-y}$, with $x=0$ (solid) and $x=0.15$ (dotted). The data curves have a common vertical scale, but are offset for clarity.

doping, and gives arguments that the gap filling must be derived by transfer of weight from both the valence and conduction bands. It is pointed out that the similar values of $E_{\mathrm{F}}$ for hole and electron doping would be implied if the metals have a Fermi surface obeying the Luttinger counting theorem [55]. This theorem states that the volume enclosed by the Fermi surface is unchanged as Coulomb interactions are turned on, so long as the symmetry of the ground state remains the same. Direct evidence for the counting theorem has been provided by high-resolution ARPES studies of the normal state [56-58]. These studies find that the near- $E_{\mathrm{F}}$ emission for $\mathrm{Bi}_{2} \mathrm{Sr}_{2} \mathrm{CaCu}_{2} \mathrm{O}_{8}$ disperses with $k$, and has $E_{\mathrm{F}}$ crossings nearly consistent with the Fermi surface predicted in band theory. Earlier lower 
resolution work also indicated Fermi-level crossings [48]. The near- $E_{\mathrm{F}}$ emission in $\mathrm{Nd}_{1.85} \mathrm{Ce}_{0.15} \mathrm{CuO}_{4}$ appears to be $k$-dependent [59], although $E_{\mathrm{F}}$ crossings have not been identified. The Fermi surface aspects of the gap states argue against donor-acceptor type impurity states as a primary mechanism. However, impurity and disorder effects cannot be entirely dismissed, as shown by the finding of gap states in insulating $\mathrm{Li}_{x} \mathrm{Ni}_{1-x} \mathrm{O}$ [60] and $\mathrm{Nd}_{1.95} \mathrm{Ce}_{0.05} \mathrm{CuO}_{4}$ [51].

Although the observation of the Luttinger volume is consistent with a Fermi liquid, as assumed by most ARPES workers cited here, it is also consistent with the marginal Fermi liquid [61] and the Luttinger liquid [62]. The latter is an elegant scenario in which there is a Fermi surface that obeys the Luttinger counting theorem, but where there is otherwise not a Fermi liquid because the electron is a composite of independent charge and spin excitations called holons and spinons. The electron removal lineshape is expected to differ from that of the Fermi liquid because it reflects the dynamics of the holon and spinon produced when the electron is removed. The analysis of experimental lineshapes entails technical issues beyond the scope of the discussion here.

I conclude with an argument that the Luttinger liquid scenario leads to the occurrence of $E_{\mathrm{F}}$ in the insulator gap. From a spectroscopic viewpoint, the metal appears to be formed by transferring a small amount of the insulator's singleparticle spectral weight to low-lying intrinsic excitations. In the insulator the electronic excitations at the ground state are the magnons, which do not couple to single-particle spectral weight. But in the metal the spin degrees of freedom take the form of spinons, which have the energetics of the insulator magnons, but are fermions. I propose to think of the near- $E_{F}$ emission as a 'hybridization' of insulator singlespectral spectral weight to the low-lying spin degrees of freedom, made possible by the change from magnons in the insulator to spinons in the metal, a profound change in statistics, but not energetics. The resulting weight and metal $E_{\mathrm{F}}$ should lie in the insulator gap, but with most of the weight remaining at higher energies characteristic of the insulator, as observed.

\section{Acknowledgements}

Research support by the U.S. National Science Foundation through the Low Temperature Physics Program, Grant No. DMR-87-21654 is gratefully acknowledged. This paper was written while I was a visitor at the Max-Planck Institut für Festkörperforschung during the summer of 1990. I thank Prof. Drs. O.K. Andersen and P. Fulde and Dr. O. Gunnarsson for hospitality during the visit, and gratefully acknowledge the Alexander von Humboldt-Stiftung of the Federal Republic of Germany for supporting the visit through a Senior U.S. Scientist Award.

\section{References}

[1] Narrow-Band Phenumena, J.C. Fuggle, G.A. Sawatzky and J.W. Allen, eds. (Plenum, New York, 1988).

[2] J.W. Allen, S.-J. Oh, O. Gunnarsson, K. Schönhammer, M.B. Maple, M.S. Torikachvili and I. Lindau, Adv. Phys. 35 (1986) 275.

[3] J.W. Allen, in: Synchrotron Radiation Research: Advances in Surface Science, R.Z. Bachrach, ed. (Plenum, New York, 1991) ch. 7, in press.

[4] D.D. Sarma, F.U. Hillebrecht, C. Carbone and A. Zangwill, Phys. Rev. B 36 (1987) 2916.

[5] A.J. Arko, D.D. Koelling, C. Capasso, M. del Giudice and C.G. Olson, Phys. Rev. B 38 (1988) 1627.

[6] J.W. Allen, J. Magn. \& Magn. Mater. 76 \& 77 (1988) 324.

[7] J.W. Allen, J.-S. Kang, L.-Z. Liu, O. Gunnarsson, N.E. Christensen, O.K. Andersen, M.B. Maple, M.S. Torikachvili, D.D. Koelling, W.P. Ellis, B.B. Pate, Z.-X. Shen, J.J. Yeh and I. Lindau, Phys. Scr. T31 (1990) 232.

[8] J.W. Allen and R.M. Martin, Phys. Rev. Lett. 49 (1982) 1106.

[9] O. Gunnarsson and K. Schönhammer, in: Handbook on the Physics and Chemistry of Rare Earths, Vol. 10, K.A. Gschneidner Jr., L. Eyring and S. Hüfner, eds. (North-Holland, Amsterdam, 1987) p. 103.

[10] N.E. Bickers, D.L. Cox and J.W. Wilkins, Phys. Rev. Lett. 36 (1987) 2036.

[11] S.J. Oh, S. Suga, A. Kakizaki, M. Taniguchi, T. Ishii, J.-S. Kang, J.W. Allen, O. Gunnarsson, N.E. Christ- 
ensen, A. Fujimori, T. Suzuki, T. Kasuya, T. Miyahara, H. Kato, K. Schönhammer, M.S. Torikachvili and M.B. Maple, Phys. Rev. B 37 (1988) 2831.

[12] F. Patthey, W.-D. Schneider, Y. Baer and B. Delley, Phys. Rev. Lett. 58 (1987) 2810.

[13] P. Strange and D.M. Newns, J. Phys. F 16 (1986) 335.

[14] G. Zwicknagl, J. Magn. \& Magn. Mater. 76 \& 77 (1988) 16.

[15] A.J. Freeman, B.I. Min and M.R. Norman, in: Handbook on the Physics and Chemistry of Rare Earths, Vol. 10, K.A. Gschneidner Jr., L. Eyring and S. Hüfner, eds. (North-Holland, Amsterdam, 1987) p. 165.

[16] A.K. McMahan and R.M. Martin, in: Narrow Band Phenomena, J.C. Fuggle, G.A. Sawatzky and J.W. Allen, eds. (Plenum, New York, 1988) p. 133.

[17] O. Gunnarsson, N.E. Christensen and O.K. Andersen, J. Magn. \& Magn. Mater. 76 \& 77 (1988) 30.

[18] F. Steglich, J. Aarts, C.D. Bredl, W. Lieke, D. Meschede, W. Franz and H. Schäfer, Phys. Rev. Lett. 43 (1979) 1892.

[19] F. Steglich, C.D. Bredl, W. Lieke, U. Rauchschwalbe and G. Sparn, Physica B 126 (1984) 82.

[20] J.-S. Kang, J.W. Allen, O. Gunnarsson, N.E. Christensen, O.K. Andersen, Y. Lassailly, M.B. Maple and M.S. Torikachvili, Phys. Rev. B 41 (1990) 6610.

[21] R.D. Parks, M.L. denBoer, S. Raaen, J.L. Smith and G.P. Williams, Phys. Rev. B 30 (1984) 1580.

[22] S. Horn, E. Holland-Moritz, M. Liewenhaupt, F. Steglich, H. Scheuer, A. Benoit and J. Flouquet, Phys. Rev. B 23 (1981) 3121.

[23] O. Gunnarsson and N.E. Christensen, Phys. Rev. B, in press.

[24] O. Gunnarsson and K. Schönhammer, Phys. Rev. B 40 (1989) 4160.

[25] Y. Baer, in: Handbook on the Physics and Chemistry of the Actinides, Vol. 1, A.J. Freeman and G.H. Lander, eds. (North-Holland, Amsterdam, 1984) ch. 4, p. 271.

[26] Y. Baer, H.R. Ott and K. Andres, Solid State Commun. 36 (1980) 387.

[27] B. Reihl, N. Mârtensson, D.E. Eastman, A.J. Arko and O. Vogt, Phys. Rev. B 26 (1982) 1842.

[28] A.J. Arko, D.D. Koelling, B.D. Dunlap and A.W. Mitchell, J. Appl. Phys. 63 (1988) 3680.

[29] J.-S. Kang, J.W. Allen, M.B. Maple, M.S. Torikachvili, W.P. Ellis, B.B. Pate, Z.-X. Shen, J.J. Ych and I. Lindau, Phys. Rev. B 39 (1989) 13529.

[30] C. König, Z. Phys. B 50 (1983) 33.

[31] L.-Z. Liu, J.W. Allen, M.B. Maple and M.S. Torikachvili, to be published.

[32] F.U. Hillebrecht, J.C. Fuggle, G.A. Sawatzky and R. Zeller, Phys. Rev. Lett. 51 (1983) 1187.

[33] N.F. Mott, Proc. Phys. Soc., London, Sect. A 62 (1949) 416.

[34] P.W. Anderson, Phys. Rev. 115 (1959) 2.

[35] J. Hubbard, Proc. Roy. Soc. A 276 (1963) 238.

[36] O. Gunnarsson, J.W. Allen, O. Jepsen, T. Fujiwara, O.K. Andersen, C.G. Olson, M.B. Maple, J.-S. Kang,
L.-Z. Liu, J.-H. Park, R.O. Anderson, W.P. Ellis, R. Liu, J.T. Markert, Y. Dalichauouch, Z.-X. Shen, P.A.P. Lindberg, B.O. Wells, D.S. Dessau, A. Borg, I. Lindau and W.E. Spicer, Phys. Rev. B 41 (1990) 4811.

[37] J.W. Allen, C.G. Olson, M.B. Maple, J.-S. Kang, L.Z. Liu, J.-H. Park, R.O. Anderson, W.P. Ellis, J.T. Markert, Y. Dalichaouch and R. Liu, Phys. Rev. Lett. 64 (1990) 595 .

[38] H. Takagi, S. Uchida, and Y. Tokura, Phys. Rev. Lett. 62 (1989) 1197.

[39] J.T. Markert and M.B. Maple, Solid State Commun. 70 (1989) 145.

[40] A.K. McMahan, R.M. Martin and S. Satpathy, Phys. Rev. B 38 (1988) 6650.

[41] H. Eskes and G.A. Sawatzky, Phys. Rev. Lett. 61 (1988) 1415

[42] G.A. Sawatzky and J.W. Allen, Phys. Rev. Lett. 53 (1984) 2339

[43] J. Zaanen, G.A. Sawatzky and J.W. Allen, Phys. Rev. Lett. 55 (1985) 418.

[44] J.W. Allen, J. Magn. \& Magn. Mater. 47 \& 48 (1985) 168.

[45] F.C. Zhang and T.M. Rice, Phys. Rev. B 37 (1988) 3759.

[46] Z.-X. Shen, C.K. Shih, O. Jepsen, W.E. Spicer, I. Lindau and J.W. Allen, Phys, Rev. Lett. 64 (1990) 2442.

[47] R. Böttner, N. Schroeder, E. Dietz, U. Gerhardt, W. Assmus and J. Kowalewski, Phys. Rev. B 41 (1990) 8679 .

[48] T. Takahashi, H. Matsuyama, H. Katayama-Yoshida, Y Okabe, S. Hosoya, K. Seki, H. Fujimoto, M. Sato and H. Inokuchi, Phys. Rev. B 39 (1989) 6636.

[49] A. Fujimori and F. Minami, Phys. Rev. B 30 (1984) 957.

[50] Z.-X Shen, J.W. Allen, J.J. Yeh, J.-S. Kang, W. Ellis, W. Spicer, I. Lindau, M.B. Maple, Y.D. Dalichaouch, M.S. Torikachvili, J.Z. Sun and T.H. Geballe, Phys. Rev. B 36 (1987) 8414.

[51] Y. Fukuda, T. Suzuki, M. Nagoshi, Y. Syono, K. Ohishi and M. Tachiki, Solid State Commun. 72 (1989) 1183.

[52] H. Namatame, A. Fujimori, Y. Takano, M. Nakamura, K. Yamaguchi, A. Misu, H. Matsubara, S. Suga, H. Eisaki, T. Itoh, H. Takagi and S. Uchida, Phys. Rev. B 41 (1990) 7205.

[53] H. Matsuyama, T. Takahashi, H. Katayama-Yoshida, T. Kashiwakura, Y. Okabe, S. Sato, N. Kosugi, A. Yagishita, K. Tanaka, H. Fujimoto and H. Inokuchi, Physica C 160 (1989) 567.

[54] A. Fujimori, Y. Tokura, H. Eisaka, H. Takagi, S. Uchida and M. Sato, Phys. Rev. B 40 (1989) 7303.

[55] J.M. Luttinger, Phys. Rev. 119 (1960) 1153.

[56] C.G. Olson, R. Liu, A.-B. Yang, D.W. Lynch, A.J Arko, R.S. List, B.W. Veal, Y.C. Chang, P.Z. Jiang and A.P. Paulikas, Phys. Rev. B (1990), in press.

[57] R. Manzke, T. Buslaps, R. Claessen, M. Skibowski and J. Fink, Physica C 152-154 (1989) 1381. 
[58] J.C. Campuzano, G. Jennings, M. Faiz, L. Beaulaigue, B.W. Veal, J.Z. Liu, A.P. Paulikas, K. Vandervoort, H. Claus, R.S. List, A.J. Arko and R.J. Bartlett, Phys. Rev. Lett. 64 (1990) 2308.

[59] Y. Sakisaka, T. Maruyama, Y. Morikawa, H. Kato, K. Edamoto, M. Okusawa, Y. Aiura, H. Yanashima, T. Terashima, Y. Bando, K. Iijima, K. Yamasoto and K. Hirata, Solid State Commun. 74 (1990) 609.
[60] P. Kuiper, G. Kruizinga, J. Ghijsen, G. A. Sawatzky and H. Verweij, Phys. Rev. Lett. 62 (1989) 221;

$J$. van Elp, private communication.

[61] C.M. Varma, P.B. Littlewood, S. Schmidt-Rink, E. Abrahams and A.E. Ruckenstein, Phys. Rev. Lett. 63 (1989) 1996.

[62] P.W. Anderson, Phys. Rev. Lett. 64 (1990) 1839. 